\section{Ethics and Digital Imaging}

\section{J. M. Mackenzie, M. G. Burke, T. Carvalho and A. Eades MSA SubCommittee on the Ethics of Digital Imaging john_mackenzie@ncsu.edu}

The amazing growth of digital imaging in the past several years has blurred the line between "real" images and those that are digitally enhanced. In most of our movies and print advertisements, we see amazing effects that create images that look real but are not.

For the last several decades film has been the predominant method that scientists employ to record the images viewed through their microscopes. Film has been readily accepted as a valid, archival recording medium because it was difficult to alter once the exposure was made and the film or print developed. The improvements in computer power and image resolution, coupled with environmental considerations, have spurred the scientific community to replace photographic processes with digital images. Processes that took hours and days are now performed in minutes. Imaging programs like Adobe Photoshop can perform all of the same photographic steps that required a darkroom, and then can do much more. The image can be "burned" onto a CD or DVD disk which is difficult to alter. Printer technology has advanced so rapidly that inkjet prints rival photographic prints. Images are often distributed digitally and viewed on displays that continue to improve. As the hardware for digital imaging improves, the quality of digital images is approaching photographic quality for a fraction of the cost, and publication quality images are produced in a fraction of the time required for film-based photography.

The most important improvement in the image recording process is that a duplicate of the digital image is identical to the original. This fact also raises a serious concern. How can one determine if an image is the original? That question is not easily answered.

The ease of use and the ability to manipulate the images so easily has led many microscopists to question what a "proper" manipulation is and what is "improper". Several years ago, this question was asked of the Microscopy Society of America. A subcommittee was formed with John Mackenzie, Grace Burke, and Tina Carvalho as members. The report of that subcommittee is the basis of this article. We felt that a more general audience might wish to examine the ethics statement and to see the general discussion that led to that statement.

There is widespread and growing use of digital imaging in the microscopy field, both in the life and physical sciences. The photographic plates/film produces a "real" image that is simply a recording of the amount of light or electrons that interact with the emulsion. The elimination of the "real" image and its replacement by a digital image file, which can be manipulated, provides a serious concern about responsible/ethical image manipulation and reporting. Film-based microscopy has been viewed as unimpeachable data for the most part - after all, film-derived micrographs are frequently used as evidence in scientific and legal investigations.

It is therefore important for scientists to consider how much - if any - image manipulation can be performed on the acquired digital image before it no longer represents the "same" data as the original image. What is the proper documentation? Are these image manipulation operations traceable? As with any ethical question, each answer produces a large number of other questions. Let us try to narrow the focus a bit.
The subcommittee was charged with two tasks: 1) defining the problem under consideration; and 2) creating an official MSA Policy Statement on the Ethics of Digital Image Processing.

There is considerable discussion and interest in digital imaging and subsequent processing among the microscopy community. However, analysis of the discussion points raised has permitted the identification of four specific topics that are frequently grouped together under the umbrella of ethics of digital image processing. These are:

1. Ethics of Digital Imaging

2. Good practice for capture, manipulation and storage of digital images

3. Legal ownership/copyright

4. Legal and regulatory chain of evidence documentation.

It is the objective of this report to address item 1 at this time.

Scientists who use images must define what processing or image manipulation is acceptable as a representation of an unprocessed, original, true image, and what is not. This is defined as the ethics of image manipulation/processing. Ethics of Digital Imaging is "what can we ethically do to the image before we cross the imaginary line between improving it and corrupting the original without reporting it as image processing." The baseline for comparison is conventional film-based microscopy. Figure 1 contains a schematic flow diagram illustrating the film-based and digital process routes.

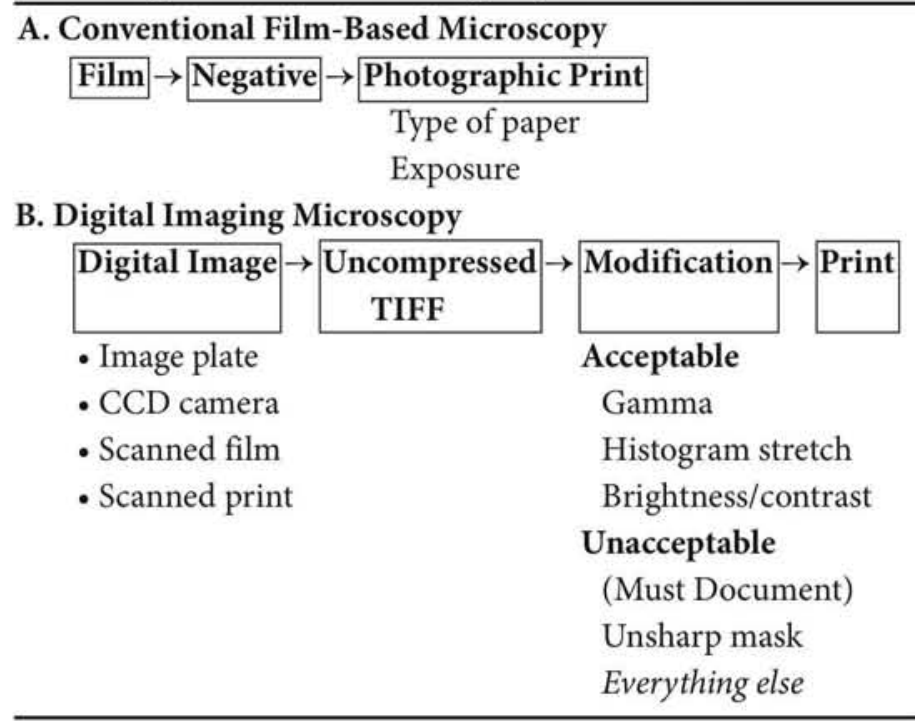

At first, the subcommittee thought that it was possible to simply take the processes used in photography and simply state that these should form the basis for digital imaging. That idea was quickly abandoned because there appeared to be no real consensus as to how photographic processes were reported. However, it was pointed out that almost all "standard" photographic operations were unacceptable with diffraction data. With this insight, the committee had to return to a simpler definition of scientific data and build from there.

Scientific experimentation is defined as testing an hypothesis with experiments that produce reproducible data. In order to publish scientific data, the methodology and experimental steps taken to achieve the experimental results must be identified. A reproducible experiment is one that another individual can duplicate based upon the reported information that will yield the same data as the original. It is recognized, however, that interpretation of those results may 
vary. Data interpretation is dependent upon the knowledge base at that time, and will usually change with time as that knowledge base increases. "Good" data are data that are reproducible and unrelated to the subsequent interpretation.

By applying the same criteria to digital imaging, this discussion is greatly simplified. The operations performed to acquire the image (including all instruments and programs used to produce and acquire the image) must be sufficiently documented to permit independent confirmation. If this cannot be performed, the image can not be considered as "data." The simple adjustments of brightness, contrast, histogram stretching and gamma correction will not qualitatively or quantitatively alter the image data in a manner that would preclude duplication of the original data. In contrast, in order to reproduce the effect of ANY other image filter, documentation of the program, the program filter and specific filter parameters are necessary. If this information is not provided, duplication of the image is not possible, and the image is therefore not valid data.

For example, if an image is acquired using a scanner that (by default) has an unsharp masking filter in operation, then appropriate documentation of that digital image must include this information (as it would be impossible to scan a similar image with another scanner and get the same result). Special attention is required on the part of scientists to identify defaults that include superfluous image manipulations or to document the model and software version used for scanning to insure data reproducibility. This task is, in fact, becoming more difficult with the constant "improvements" in both hardware and software. Scientists must constantly be vigilant and not assume that image data are unprocessed. Images should be saved as an uncompressed TIFF file (the MSA approved image format).

Ethical digital imaging and processing requires that digital image acquisition and manipulation are documented in sufficient detail to ensure reproducibility. The official MSA Policy is:

"Ethical digital imaging requires that the original uncompressed image file be stored on archival media (e.g., CD-R) without any image manipulation or processing operations. All parameters of the production and acquisition of this file, as well as any subsequent processing steps, must be documented and reported to ensure reproducibility.

Generally, acceptable (non-reportable) imaging operations include gamma correction, histogram stretching, and brightness and contrast adjustments. All other operations (such as Unsharp-Masking, Gaussian Blur, etc.) must be directly identified by the author as part of the experimental methodology. However, for diffraction data or any other image data that is used for subsequent quantification, all imaging operations must be reported."

Several points of this statement invite some further comment. Data should be saved on a disk that can not easily be altered. The best candidates currently are the CD-R format and an increasing number of DVD formats. Any of the standard formats that does not permit the data to be altered either intentionally or, as is the case more often, unintentionally. A disk should be written and closed. This makes the data about as permanent as film. These methods create disks that are also as fragile as film and require care in handling if they are to be the archival copy of the data. The strength of the digital approach is that an exact copy can be stored in a safe place.
There are few programs that record all of the steps in processing and those that do often use proprietary formats. Some programs use encryption to insure that other programs are not used to alter data. These approaches lead to a serious problem. Can a scientist in the future retrieve the data and read it. What happens if the company that produced it does not exist? The format needed would have to be universally accepted and open sourced to be worthwhile.

All data should be recorded before any of the processing is performed. Saving data in an unprocessed state insures that the data can be used in the future. If analysis is desired, the original data are available. If the processing steps used to obtain the final image are questioned, the original data are available for comparison to the processed image. . Even with large scientific image formats the cost of storage is vanishingly small. It, therefore, makes no sense to not save an original unprocessed and uncompressed image file. The MSA format for this storage is the TIFF file format.

\section{Microscopy $_{\text {ANO }}$ Microanalysis Table of Contents Preview Volume 12, Number 1, February 2006}

\section{YEARS OF X-RAY MAPPING}

\section{Editorial}

Charles E. Lyman

Tutorial Review: X-ray Mapping in Electron-Beam Instruments John J. Friel and Charles E. Lyman

The New X-ray Mapping: X-ray Spectrum Imaging above $100 \mathrm{kHz}$ Output Count Rate with the Silicon Drift Detector

Dale E. Newbury

Tomographic Spectral Imaging with Multivariate Statistical Analysis: Comprehensive 3D Microanalysis

Paul G. Kotula, Michael R. Keenan, and Joseph R. Michael

\section{MICROANALYSIS}

Win X-ray: A New Monte Carlo Program that Computes X-ray Spectra Obtained with a Scanning Electron Microscope

Raynald Gauvin, Eric Lifshin, Hendrix Demers, Paula Horny, and Helen Campbell

Beam-Induced Damage to Thin Specimens in an Intense Electron Probe

Raymond F. Egerton, Feng Wang, and Peter A. Crozier

EBSD Image Quality Mapping

Stuart I. Wright and Matthew M. Nowell

Misorientation Mapping for Visualization of Plastic Deformation via

Electron Back-Scattered Diffraction

L.N. Brewer, M.A. Othon, L.M. Young, and T.M. Angeliu

CALENDAR OF MEETINGS AND COURSES

Indexed in Chemical Abstracts, Current Contents, BIOSIS, and MEDLINE (PubMed)

MSA members receive both Microscopy Today and Microscopy and Microanalysis FREE! 